\title{
Work of the New Dynamic Meteorology Project at Columbia University
}

\section{R. PfEFfER}

The Dynamic Meteorology Project at Columbia University was established in 1959 for the purpose of studying from a physical point of view the operation of the general circulation, the development of cyclonic and anticyclonic circulations and the mechanism of wave propagation of all scales through the atmosphere. Much of this research involves the application of numerical methods of integration and the use of high-speed electronic computers. In the present talk, certain aspects of the work at Columbia during the first year will be discussed. The research to be described is intended to serve as background for subsequent numerical integrations. 